\title{
Stability Analysis of WONDY (A Hydrocode Based on the Artificial Viscosity Method of von Neumann and Richtmyer) for a Special Case of Maxwell's Law*
}

\author{
By D. L. Hicks
}

\begin{abstract}
The artificial viscosity method of von Neumann and Richtmyer was originally designed and analyzed for stability in the case when the material was an ideal gas. Recently a hydrocode (WONDY) based on the von Neumann-Richtmyer scheme was used in calculating wave propagation problems in materials obeying a form of Maxwell's material law; signs of an unstable difference scheme appeared. A stability analysis shows that the timestep restrictions required for stability in certain cases can be more stringent for material laws of the Maxwell type than they are for material laws of the ideal gas type.
\end{abstract}

1. Introduction. In $1950 \mathrm{~J}$. von Neumann and R. Richtmyer [5] presented their artificial viscosity method along with an approximate stability analysis for the case when the material law was the ideal gas law. In 1954 their stability analysis was improved by G. N. White [3]. In 1966 R. J. Thompson [2] presented an approximate stability analysis in the case where the stress depends only on the strain.

All of the aforementioned stability analyses were for material laws of the pointfunction type. That is, the stress at a point in the material and at a certain time is given as a function of the strain and specific internal energy at that point and time. The ideal gas law and Hooke's law are classic examples of point-function type material laws.

Recently, there has been interest in material laws that are not of the pointfunction type. For example, many materials obey a history-functional type material law [1]. That is, the stress is given as the value of a functional that depends on the history of the strain, strain rates, etc. Another way of saying this is that the stress is prescribed in terms of a differential equation rather than by a point-function. That is, a differential equation for the stress rate is given in which the stress rate is related to the strain rate with coefficients which may depend on the stress, strain, etc. The classic example is Maxwell's material law. Material laws of this type are often referred to as rate dependent material laws.

There are many different forms of rate dependent material laws. In this brief paper the first results of stability analyses of hydrocodes with rate dependent material laws are presented. A simple but representative law was chosen for the first stability

Received January 31, 1978.

AMS (MOS) subject classifications (1970). Primary $65 \mathrm{M10.}$

* This work was supported by the Department of Energy. 
analysis; it is a special case of Maxwell's material law. In the case of this simple law a stability analysis indicates that timestep restrictions can be more severe for rate dependent laws.

\section{Notation and Nomenclature.}

Conservation Laws. The one-dimensional, Lagrangean conservative form of the laws of conservation of volume, momentum, and energy are expressed by

$$
\partial \mathbf{U} / \partial t+\partial \mathbf{F} / \partial \mu=0
$$

where

$$
\mathbf{U}=\left(\begin{array}{c}
V \\
u \\
E
\end{array}\right) \quad \text { and } \quad \mathbf{F}=\left(\begin{array}{c}
-u \\
\sigma \\
u \sigma
\end{array}\right)
$$

Here $t$ is time; $\mu$ is material coordinate; $V$ is specific volume; $V=1 / \rho$, where $\rho$ is mass density; $u$ is specific momentum; $E$ is specific total energy; $E=\boldsymbol{E}+1 / 2 u^{2}$,

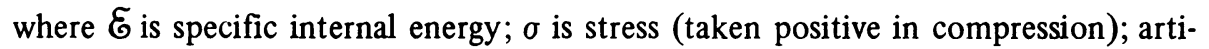
ficial viscosity will be denoted $q$; strain is given by $c=1-V / V^{0}$, where $V^{0}$ is the initial value of $V$.

Material Laws. If the stress depends only on the strain, the material law is called a mechanical equation of state (e.g. Hooke's law). If the stress also depends on temperature, or internal energy, or entropy, the material law is called a thermodynamic equation of state (e.g. ideal gas law). Both the mechanical equation of state and the thermodynamic equation of state are examples of the point-function type material law.

An example of material laws of the history-functional type is Maxwell's material law (see [1])

$$
\partial \sigma / \partial t+a^{2} \partial V / \partial t+R=0
$$

where $a$ is the acoustic impedance and $R$ is the relaxation function. In Maxwell's material law $a$ and $R$ are allowed to depend on $\sigma$ and $V$. For many materials $R$ is of the form $\left(\sigma-\sigma_{\mathrm{eq}}\right) / \tau$, where $\sigma_{\mathrm{eq}}$ is the equilibrium stress and $\tau$ is the relaxation time of the material.

Discrete Notation. Let $\mu_{j}=j \Delta \mu$ and $t^{n}=n \Delta t$ where $\Delta \mu$ and $\Delta t$ are the material and time increments. The approximation to $f\left(\mu_{j}, t^{n}\right)$ is denoted $f_{j}^{n}$. The CFL (Courant, Friedrichs, Lewy) number (see [4]) is given by $\alpha=a \Delta t / \Delta \mu$. Differences with respect to $\mu$ and $t$ are denoted $\Delta$. and $\Delta^{*}$, respectively. For example, $\Delta . f_{j+1 / 2}^{n}$ $\equiv f_{j+1}^{n}-f_{j}^{n}$ and $\Delta^{\cdot} f_{j}^{n+1 / 2} \equiv f_{j}^{n+1}-f_{j}^{n}$.

3. Background. Since about 1950 the von Neumann-Richtmyer (VNR) scheme has been the basis for many hydrocodes. The stability analyses appear mainly in laboratory reports; therefore, a brief history of those analyses is presented here. First, in the original paper [5] von Neumann and Richtmyer did an approximate stability analysis (in the case of the ideal gas law) by considering separately the shock regions and normal regions. (See also [4, pp. 320-324].) For normal regions their analysis 
yielded the famous CFL condition; i.e.,

$$
\alpha \leqslant 1
$$

One can show that

$$
\alpha<1
$$

is necessary and sufficient for stability of the VNR scheme when $q=0$ and the material law is Hooke's law in one dimension, i.e.,

$$
\sigma=a^{2} V^{0} e .
$$

(This follows from results on p. 263 of [4].) For shock regions von Neumann and Richtmyer's approximate stability analysis produced the restriction

$$
4(\Lambda / a) \alpha \leqslant 1
$$

where $\Lambda$ is the coefficient of the artificial viscosity; i.e.,

$$
q=-\Lambda \Delta \cdot u
$$

The original VNR viscosity was given by

$$
\Lambda=c_{2} \rho|\Delta \cdot u|,
$$

where $c_{2}$ is a dimensionless constant $\approx 1$. In $1954 \mathrm{H}$. G. Kolsky reported (see p. 13 of [3] ) a result of G. N. White on the stability analysis of the VNR scheme; i.e.,

$$
\alpha^{2}+4(\Lambda / a) \alpha \leqslant 1
$$

The analysis leading to (3.6) was never published [6]. White's inequality (3.6) was also reported on p. 350 of [4], unfortunately with a typographical error; the 2 exponent of $\alpha$ is missing there. In $1966 \mathrm{R}$. J. Thompson [2] did an approximate stability analysis for a mechanical equation of state in the case where $\Lambda$ is a nonnegative constant and arrived at the restriction

$$
\alpha^{2}+2(\Lambda / a) \alpha \leqslant 1
$$

as a necessary condition for stability. One can prove that (3.7) is necessary for stability when $\Lambda$ is a nonnegative constant, and the material law is Hooke's law in one dimension (3.2). This follows from Result \#1 of Section 5. The reason for the $2-4$ discrepancy in (3.7)-(3.6) is because White was doing a stability analysis with a viscosity quadratic in $\Delta . u$ (the original VNR viscosity), and that results in an extra factor of 2 in the first variation of $q$; Thompson's analysis is for a viscosity linear in $\Delta . u$.

4. Lemmas. In Section 5 certain 2 by 2 amplification matrices arise and their eigenvalues are roots of certain quadratic equations; i.e.,

$$
\lambda^{2}-2 B \lambda+C=0 \text {. }
$$

The lemmas in this section are used to arrive at the timestep restrictions of Section 
5. The proofs of Lemmas $1 \mathrm{~A}, 1 \mathrm{~B}$, and 2 are left to the reader. The proofs of Lemmas $3 \mathrm{~A}$ and $3 \mathrm{~B}$ may be found in [4].

LEMmA 1A. Let $B$ and $C$ be real numbers; $D=B^{2}-C ; \lambda_{ \pm}=B \pm D^{1 / 2} ;|\lambda|_{\max }$ $=\max \left|\lambda_{ \pm}\right|$.

Case (a): If $D \geqslant 0$ and $B^{2}>1$, then

$$
|\lambda|_{\max }>1 \text {. }
$$

Case (b): If $D \geqslant 0$ and $B^{2} \leqslant 1$, then

$$
\left[|\lambda|_{\max } \leqslant 1 \text { iff } 2|B| \leqslant C+1\right] .
$$

Case (c): If $D<0$, then

$$
\left[|\lambda|_{\max } \leqslant 1 \text { iff } C \leqslant 1\right] .
$$

Moreover, the result also holds when the $\leqslant$ signs inside the square brackets are replaced by $<$ or $=$.

Lemma 1B. Let $B=1-b ; C=1-c ; 2 b \geqslant c \geqslant 0$. Then

$$
\left[|\lambda|_{\max } \leqslant 1 \text { iff } 2 b+c \leqslant 4\right] \text {. }
$$

Lemma 2. Assume $A$ real, $B$ and $\alpha$ positive; let $D=B^{2}+A$; and let $\alpha^{\prime}=$ $\alpha\left(B+D^{1 / 2}\right)$. Consider the following inequalities

$$
\left[A \alpha^{2}+2 B \alpha \leqslant 1\right]
$$

and

$$
\left[\alpha^{\prime} \leqslant 1\right]
$$

Case (a): If $D \geqslant 0$, then (4.1) if and only if (4.2).

Case (b): If $D<0$, then (4.1) holds for all $\alpha$.

Lemma 3A (Von NeumanN'S NeCESSARy CONDITION). Let $\mathbf{G}(\Delta t, k)$ be the amplification matrix for the Fourier component of index $k$; let $|\lambda|_{\max }$ be its spectral radius. Then

$$
|\lambda|_{\max } \leqslant 1+O(\Delta t)
$$

is a necessary condition for stability.

LEMMA 3B (ONE OF RICHTMYER'S SUFFICIENT CONDITIONS). Let $\Delta$ be the determinant of the normalized eigenvectors of $\mathbf{G}$. If there exists a constant $\delta$ such that $|\Delta| \geqslant \delta>0$ (uniformly in $(\Delta t, k)$ ), then the von Neumann condition is sufficient for stability.

5. Results. The stability analysis is to be done for the material law given by

$$
\frac{\partial \sigma}{\partial t}+a^{2} \frac{\partial V}{\partial t}+\frac{\sigma-\sigma_{\mathrm{eq}}}{\tau}=0
$$

where $a, \sigma_{\mathrm{eq}}$, and $\tau$ are constants with $a$ and $\tau$ positive.

In addition to (5.1) the equations 


$$
\partial V / \partial t=\partial u / \partial \mu
$$

and

$$
\partial u / \partial t=-\partial \sigma / \partial \mu
$$

are needed to complete the system. (Note that the conservation of energy equation is not needed.) WONDY's difference scheme for (5.3) is

$$
\left(\frac{\Delta u}{\Delta t}\right)_{j}^{n}=-\left(\frac{\Delta \sigma}{\Delta \mu}\right)_{j}^{n}-\left(\frac{\Delta q}{\Delta \mu}\right)_{j}^{n-1 / 2}
$$

where

$$
\left(\frac{\Delta u}{\Delta t}\right)_{j}^{n}=\frac{u_{j}^{n+1 / 2}-u_{j}^{n-1 / 2}}{t^{n+1 / 2}-t^{n-1 / 2}}, \quad\left(\frac{\Delta \sigma}{\Delta \mu}\right)_{j}^{n}=\frac{\sigma_{j+1 / 2}^{n}-\sigma_{j-1 / 2}^{n}}{\mu_{j+1 / 2}-\mu_{j-1 / 2}},
$$

and $q$ is the artificial viscosity with

and

$$
\left(\frac{\Delta q}{\Delta \mu}\right)_{j}^{n-1 / 2}=\frac{q_{j+1 / 2}^{n-1 / 2}-q_{j-1 / 2}^{n-1 / 2}}{\mu_{j+1 / 2}-\mu_{j-1 / 2}}
$$

$$
q_{j+1 / 2}^{n-1 / 2}=-\Lambda \Delta \cdot u_{j+1 / 2}^{n-1 / 2}
$$

with

$$
\Delta \cdot u_{j+1 / 2}^{n-1 / 2}=u_{j+1}^{n-1 / 2}-u_{j}^{n-1 / 2} .
$$

$\Lambda$ is always required to be nonnegative and in general it may be rather complicated (see [4] and [2]). For simplicity in the following analysis, $\Lambda$ is taken to be constant. WONDY's difference equation for (5.2) is

$$
\left(\frac{\Delta V}{\Delta t}\right)_{j+1 / 2}^{n+1 / 2}=\left(\frac{\Delta u}{\Delta \mu}\right)_{j+1 / 2}^{n+1 / 2}
$$

where

$$
\left(\frac{\Delta V}{\Delta t}\right)_{j+1 / 2}^{n+1 / 2}=\frac{V_{j+1 / 2}^{n+1}-V_{j+1 / 2}^{n}}{t^{n+1}-t^{n}}
$$

and

$$
\left(\frac{\Delta u}{\Delta \mu}\right)_{j+1 / 2}^{n+1 / 2}=\frac{u_{j+1}^{n+1 / 2}-u_{j}^{n+1 / 2}}{\mu_{j+1}-\mu_{j}} .
$$

Finally WONDY's difference equation for (5.1) is

$$
\left(\frac{\Delta \sigma}{\Delta t}\right)_{j+1 / 2}^{n+1 / 2}+a^{2}\left(\frac{\Delta V}{\Delta t}\right)_{j+1 / 2}^{n+1 / 2}+\frac{\sigma_{j+1 / 2}^{n}-\sigma_{\mathrm{eq}}}{\tau}=0
$$

and from (5.6), letting $h=\Delta t / \tau$, follows

$$
\Delta^{\cdot} \sigma_{j+1 / 2}^{n+1 / 2}+a^{2} r \Delta \cdot u_{j+1 / 2}^{n+1 / 2}+h\left(\sigma_{j+1 / 2}^{n}-\sigma_{\mathrm{eq}}\right)=0,
$$

where $r=\Delta t / \Delta \mu$. 
Remark. Observe that if $h=0$, then (5.7) is satisfied by Hooke's law in one dimension (3.2).

In (5.4) and (5.7) replace $u_{j}^{n+1 / 2}$ by $v^{n+1} \xi^{j}$ and $\sigma_{j+1 / 2}^{n}-\sigma_{\text {eq }}$ by $-a w^{n} \xi^{j+1 / 2}$ where $\xi=\exp (i k \Delta \mu)$ and let

$$
\mathbf{U}^{n}=\left[\begin{array}{c}
v^{n} \\
w^{n}
\end{array}\right]
$$

to get

$$
\mathbf{U}^{n+1}=\mathbf{G}(\Delta t, k) \mathbf{U}^{n}
$$

where

$$
\mathbf{G}(\Delta t, k)=\left[\begin{array}{cc}
1-\gamma & i \beta \\
i \beta(1-\gamma) & 1-\beta^{2}-h
\end{array}\right]
$$

with

$$
\alpha=a r
$$

and

$$
\beta=2 \alpha \sin (k \Delta \mu / 2)
$$

and

$$
\gamma=\Lambda r(\beta / \alpha)^{2}
$$

Note that

$$
\operatorname{det}(\mathbf{G}-\lambda \mathbf{I})=\lambda^{2}-2 B \lambda+C,
$$

where

$$
B=1-\left(\beta^{2}+\gamma+h\right) / 2
$$

and

$$
C=(1-\gamma)(1-h)
$$

Remark. Note that (5.8) agrees with Richtmyer and Morton [4, p. 262], when $h=0=\Lambda$.

RESULT \#1. If $h=0$ and $\Lambda \geqslant 0$, then

$$
\alpha^{2}+2 \Lambda r \leqslant 1
$$

is necessary for the stability of $\mathbf{G}(\Delta t, k)$ given by (5.8).

Proof Sketch. Apply Lemma 1B with $2 \mathrm{~b}=\beta^{2}+\gamma$ and $c=\gamma$ to get $\beta^{2}+2 \gamma$ $\leqslant 4$ and (5.12) follows. End of proof sketch.

Remark. Note that (5.12) is Thompson's inequality.

RESULT \#2. Let $h=0$ and $\Lambda \geqslant 0$; also let $\alpha^{\prime}=a^{\prime} \Delta t / \Delta \mu$, where

$$
a^{\prime}=a\left\{\frac{\Lambda}{a}+\left[\left(\frac{\Lambda}{a}\right)^{2}+1\right]^{1 / 2}\right\}
$$


Then a necessary condition for stability of the $\mathbf{G}$ given by (5.8) is

$$
\alpha^{\prime} \leqslant 1
$$

Proof Sketch. Apply Lemma 2 to Result \#1. End of proof sketch.

Remark. The timestep restriction enforced in WONDY is $\alpha^{\prime} \leqslant \theta<1$, where $\theta$ is usually taken to be 0.9 .

Result \#3. If $0 \leqslant h<1$ and $0 \leqslant \Lambda r<1 / 4$, then

$$
\alpha^{2}+h(1 / 2-\Lambda r)+2 \Lambda r \leqslant 1
$$

is necessary for stability of the G given by (5.8).

Proof Sketch. Note that

$$
2 b+c=\beta^{2}+2(\gamma+h)-\gamma h
$$

and apply Lemma $1 B$ to get $|\lambda|_{\max } \leqslant 1$ if and only if (5.15). Thus, necessity follows from Lemma 3A. End of proof sketch.

Remark. Note that if $\Lambda=0$, then (5.15) reduces to

$$
\alpha^{2}+h / 2 \leqslant 1 \text {. }
$$

Therefore, it is necessary for stability that $h<2$.

RESULT \#4. If $0 \leqslant h<1$ and $0 \leqslant \Lambda r<\alpha(1-\alpha)+f(h)$ where $f(h)=$ $\alpha\left[(1-h)^{1 / 2}-1\right]+h / 4$, then

$$
\alpha^{2}+h(1 / 2-\Lambda r)+2 \Lambda r<1
$$

is sufficient for the stability of $\mathbf{G}$ given by (5.8).

Proof Sketch. First consider the case when $(\sin k \Delta \mu / 2)^{2}<h K$ where $K$ is a constant to be determined later. Then $\mathbf{G}=\mathbf{I}+O(h)$ which is sufficient for stability.

Next consider the case when $(\sin k \Delta \mu / 2)^{2} \geqslant K h$. Let $D=B^{2}-C ; \lambda_{ \pm}=B \pm$ $D^{1 / 2} ; \rho_{ \pm}^{2}=\beta^{2}+\left|1-\gamma-\lambda_{ \pm}\right|^{2} ; v_{1}^{ \pm}=-i \beta / \rho_{ \pm}$and $v_{2}^{ \pm}=\left(1-\gamma-\lambda_{ \pm}\right) / \rho_{ \pm}$. Note that $\left\{\mathbf{v}^{+}, \mathbf{v}^{-}\right\}$is a complete set of normalized eigenvectors of $\mathbf{G}$. Observe that

$$
\left|\operatorname{det}\left(\mathbf{v}^{+}, \mathbf{v}^{-}\right)\right|^{2}=4 \beta^{2}|D| /\left(\rho_{+} \rho_{-}\right)^{2} \text {. }
$$

The idea is to show there exists a $\delta>0$ such that

$$
4 \beta^{2}|D| \geqslant \delta\left(\rho_{+} \rho_{-}\right)^{2} ;
$$

then apply Lemma 3B. The next step is to show that (5.16) implies $D<0$ when $(\sin k \Delta \mu / 2)^{2} \geqslant K h$ and $0 \leqslant \Lambda r<\alpha(1-\alpha)+f(h)$. Note that

$$
D=-\beta^{2}\left\{1-g\left(\beta^{2}\right)\right\} \text {, }
$$

where

$$
\beta^{2} g\left(\beta^{2}\right)=\left(\left(\beta^{2}+\gamma+h\right) / 2\right)^{2}-\gamma h .
$$

It shall be shown that $g\left(\beta^{2}\right)<1$ for $K h \leqslant(\beta / 2 \alpha)^{2} \leqslant 1$. Note that $g$ is monotone increasing for $\beta^{2} \geqslant h /\left(1+\Lambda r / \alpha^{2}\right)$. If the constant $K$ is chosen such that $4 K\left(\alpha^{2}+\Lambda r\right)$ $\geqslant 1$, then the largest value of $g$ on the interval $\left[4 \alpha^{2} K h, 4 \alpha^{2}\right]$ occurs at $\beta^{2}=4 \alpha^{2}$. 
Therefore, let us so choose $K$. Now considering $g\left(4 \alpha^{2}\right)<1$ as a restriction on $\Lambda r$ is equivalent to $\Lambda r<\alpha(1-\alpha)+f(h)$. (Use Lemma 2, Case (a) on the quadratic in $\gamma$.) This establishes $D<0$. When $D<0$, then

$$
\rho_{ \pm}^{2}=\beta^{2}|2-\gamma|
$$

and (5.17) reduces to

$$
1-g\left(\beta^{2}\right) \geqslant \delta(2-\gamma)^{2} / 4 .
$$

Hence, (5.17) requires $g\left(\beta^{2}\right)<1$; this has already been shown. End of proof sketch.

Remarks. As an aid to the reader, an explicit verbal statement of the foregoing results is now provided. Result \#1 proves that Thompson's inequality is necessary in the case of difference equations (5.4), (5.5 with $\Lambda$ constant), (5.6), and (5.7 with $h$ $=0$ ) (in other words when the material law is Hooke's law with viscosity linear in $\Delta . u$ ). Result \#2 reduces the quadratic stability inequality of Result \#1 to a linear inequality. Result \#3 shows necessary and Result \#4 shows sufficient conditions for stability when $0 \leqslant h<1$.

6. Concluding Remarks. Inequality (5.12) is essentially the inequality that WONDY enforces. Note that for calculations in which the ratio $h=\Delta t / \tau$ (where $\tau$ is the relaxation time of the material) is large that (5.12) might be satisfied while (5.15) is violated. This appears to be the reason why some of the calculations done with WONDY on rate dependent material laws have shown signs of instability.

Acknowledgements. The author wishes to express his gratitude to R. J. Thompson and T. J. Burns of Sandia Laboratories for their critical review of this paper. Also, he thanks G. N. White of Los Alamos for his help in supplying information about the history of the stability analyses of the von Neumann-Richtmyer scheme.

Sandia Laboratories

Albuquerque, New Mexico 87115

1. J. ASAY, D. HICKS \& D. HOLDRIDGE, "Comparison of experimental and calculated elastic-plastic wave profiles in LiF,” J. Appl. Phys., v. 46, 1975, pp. 4316-4322.

2. W. HERMANN, P. HOLZHAUSER \& R. THOMPSON, WONDY: A Computer Program for Calculating Problems of Motion in One Dimension, Sandia Laboratories Report SC-RR-66-601, 1966.

3. H. G. KOLSKY, A Method for the Numerical Solution of Transient Hydrodynamic Shock Problems in Two Space Dimensions, Los Alamos Laboratories Report LA-1867, 1954.

4. R. RICHTMYER \& K. MORTON, Difference Methods for Initial Value Problems, Interscience, New York, 1967.

5. J. VON NEUMANN \& R. RICHTMYER, "A method for the numerical calculation of hydrodynamic shocks," J. Appl. Phys., v. 21, 1950, pp. 232-237.

6. G. N. WHITE, private communication. 